\begin{tabular}{|c|l|}
\hline Title & $\begin{array}{l}\text { Biomechanical study on the effect of five different lumbar reconstruction techniques on adjacent-level intradiscal } \\
\text { pressure and lamina strain. }\end{array}$ \\
\hline Author(s) & Sudo, Hideki; Oda, Itaru; A bumi, Kuniy oshi; Ito, Manabu; Kotani, Y oshihisa; Minami, A kio \\
\hline Citation & Journal Of Neurosurgery:Spine, 5(2), 150-155 \\
\hline Issue Date & 2006-08 \\
\hline Doc URL & http://hdl.handle.net/2115/14612 \\
\hline Rights & $\begin{array}{l}\text { The final version of the paper was published in Journal Of Neurosurgery:Spine, 2006, volume5-2. For reuse of any of } \\
\text { the materials, including editorial copy, figures, or tables please contact the Journal of Neurosurgery at } \\
\text { jneuro@ virginia.edu }\end{array}$ \\
\hline Type & article (author version) \\
\hline File Information & JN2006-5-2.pdf \\
\hline
\end{tabular}

Instructions for use 


\title{
Biomechanical study on the effect of five different lumbar reconstruction techniques on adjacent level intradiscal pressure and lamina strain
}

\author{
Hideki Sudo, MD \\ Itaru Oda, MD \\ Kuniyoshi Abumi, MD \\ Manabu Ito, MD \\ Yoshihisa Kotani, MD \\ Akio Minami, MD
}

Department of Orthopaedic Surgery, Hokkaido University Graduate School of Medicine, Sapporo, Japan

Correspondence: $\quad$ Hideki Sudo, MD, PhD

Department of Orthopaedic Surgery

Hokkaido University Graduate School of Medicine

North-15, West-7, Kita-ku

Sapporo, Hokkaido 060-8638, Japan

Tel: 81-11-706-5934

Fax:81-11-706-6054

Email: hidekisudo@yahoo.co.jp

Key Words: spine biomechanics, adjacent segment, posterior lumbar interbody fusion, intradiscal pressure, lamina strain, kyphosis 


\begin{abstract}
Object. The objectives of this study were to compare biomechanical effects of five lumbar reconstruction models on the adjacent segment and to analyze effects of three factors: construct stiffness, sagittal alignment, and the number of the fused segments.

Methods. Non-destructive, flexion-extension tests were performed by applying pure moments on ten calf spinal specimens (L3-S1). One- (L5-6) or two- (L5-6-S1) segment posterior fusion methods were simulated. 1) one-segment posterolateral fusion; 2) one-segment posterolateral fusion with interbody fusion cages (one-segment PLIF/ posterolateral fusion); 3) two-segment posterolateral fusion; 4) two-segment PLIF/ posterolateral fusion; and 5) two-segment posterolateral fusion in kyphosis (two-segment kyphotic posterolateral fusion). The range of motion (ROM) of the reconstructed segments, intradiscal pressure and lamina strain in the upper adjacent segment (L4-5) were analyzed.

The ROM was significantly decreased in the PLIF/ posterolateral fusion models compared with the posterolateral fusion alone in both the one and two segment fusion models. If the number of fused segment is increased, the pressure and strains were also increased in the PLIF/ posterolateral fusion procedure more so than the posterolateral fusion procedure. The one-segment PLIF/ posterolateral fusion model demonstrated a reduced intradiscal pressure and lamina strain compared to the kyphotic two-segment posterolateral fusion model in spite of higher levels of initial stiffness.

Conclusions. If the number of fused levels can be reduced by correction of local kyphosis using the posterior lumbar interbody fusion procedure, this surgical planning may be valuable for reducing degenerative changes within the adjacent segment.
\end{abstract}


Key Words: spine biomechanics, adjacent segment, posterior lumbar interbody fusion, intradiscal pressure, lamina strain, kyphosis 


\section{Introduction}

Posterolateral fusion using the pedicle screw system has been widely used to treat unstable lumbar spine. However, posterolateral fusion is often unable to restore sagittal plane alignment or disc space height when the anterior column is broke down. Posterior lumbar interbody fusion (PLIF) combined with posterolateral fusion (PLIF/ posterolateral fusion) provides anterior column support, increases construct stiffness, ${ }^{3}$ restores spinal alignment, ${ }^{22}$ and provides higher fusion rates ${ }^{22}$ compared with the conventional posterolateral fusion alone.

On the other hand, degenerative changes in the adjacent levels to the spinal fusion have been well recognized. ${ }^{14,15}$ It was thought that less rigid fixation ${ }^{18}$ physiological alignment, ${ }^{13,19}$ and the use of short segment fusion ${ }^{5,17}$ might reduce progress of these degenerative changes within the adjacent segments. However, the details of these interactions or the synergistic effects of these factors are not yet fully understood. In our previous biomechanical study, ${ }^{21}$ it was demonstrated that comparing posterolateral fusion with residual kyphotic deformity, the PLIF/ posterolateral fusion treatment may lead to an even higher load on the adjacent segments because of the increased stiffness in the fixed segments even if the local kyphosis is corrected by PLIF. It was only simulated, however, a two-segment fixation model and did not investigate the effects of the number of fixed segments. The effect of a short segment fusion provided by the PLIF procedure on the adjacent segment remains unknown. Hence, it is uncertain whether any kyphotic deformity after the instrumentation has been applied is acceptable, or whether the PLIF procedure should be applied to restore the normal physiological alignment or to provide short segment fusion. No clinical or biomechanical reports have addressed these issues.

The objectives of this study were to compare the biomechanical effects of five different posterior lumbar reconstruction models on the adjacent segments and to analyze the effects of 
three specific factors; construct stiffness, sagittal alignment, and the number of the fused segments. 


\section{Materials and Methods}

\section{Specimens and Experimental Setup}

Ten specimens of calf cadaver lumbar spines (from the third lumbar vertebra to the sacrum, of 8-10 week-old calves) were tested. To exclude specimens with abnormalities or degenerative changes, anteroposterior and lateral radiographs were taken before testing. The soft tissues were removed taking care not to damage the disc, ligaments, or joint capsules. The upper half of the L3 vertebra and the lower half of the sacrum were cast in polyester resin molds (Flexible Body Filler of Polyester Resin, Soler Co., Ltd., Tokyo, Japan) that were reinforced by three screws $3 \mathrm{~mm}$ in diameter. The sacrum was then securely fastened onto a testing table. Radiographs were taken in each case to ensure that the L4-5 intervertebral disc was horizontally oriented. All tests were performed at room temperature. The specimens were wrapped in saline-solution-soaked towels to prevent dehydration of the tissue.

Non-destructive, biomechanical testing was performed by applying two types of pure moments

to the specimens in a previously reported manner (Figure 1). ${ }^{1,18,20,21}$ The flexion and extension moments ranged from 0 to $6 \mathrm{~N}$-m, which include the ranges of moments used in previous biomechanical studies using lumbar reconstruction models. ${ }^{1,9,10,18,21}$ As pilot studies have previously demonstrated that load-displacement and load-strain or pressure data after the second load-unload cycle were almost identical to the second, the loading procedure involved the application of a three load-unload cycle. The third loading cycle was used for data analysis of all biomechanical parameters. The specimen was allowed to creep for 30 seconds between load application and data collection. $9,10,18,21$

One- (L5-6) or two- (L5-6-S1) segment posterior fusion methods were simulated. A displacement gauge (Model 25C-20, MTS Systems, Inc. Minneapolis, MN) spanning the 
operative segment was utilized to quantify segmental displacement ${ }^{18,21}$ (if two-segment fusion was simulated, the gauge was placed over the whole fusion length of L5 to S1). The displacement gauge was attached longitudinally, bridging the anterior aspects of the vertebral bodies through the anterior longitudinal ligament. The gauge was consistently oriented at vertical direction of the segment. The range of motion (ROM) was measured with respect to the corresponding anterior longitudinal displacement $(\mathrm{mm})$ in order to evaluate the stability of the fused segments. ${ }^{18,21} \mathrm{~A}$ pressure needle transducer (R. Denton, Inc., Rochester Hills, MI) was inserted into the L4-5 intervertebral discs to obtain intradiscal pressure and investigate the biomechanical effects on the superior adjacent segment. ${ }^{6,21}$ The needle was $2.1 \mathrm{~mm}$ in diameter. Its tip housed a waterproof-coated single-strain gauge containing a 1.5-mm-diameter sensing area. The needle was initially calibrated to $1.36 \mathrm{MPa}$ with a hydraulic test. The pressure needle was inserted from the lateral side of the L4-5 intervertebral disc into the center of the nucleus pulposus. ${ }^{6,21}$ The insertion point and depth of the needle was controlled using both anterior-posterior and lateral radiographs. Uniaxial surface-strain gauges (KFG-02-120-C1-11L3M2R; Kyowa Dengyo Co., Tokyo, Japan) were sagittally mounted on the bilateral L4 lamina just craniad to the L4-L5 facet joint by using cyanoacrylate. ${ }^{4,19,21}$ The lamina strains indirectly indicated the load transmission through the posterior column of the adjacent segment. ${ }^{19}$ For the lamina strain analysis, the mean values of right and left gauges were calculated to minimize the effects of coupled motions. ${ }^{21}$

Data were acquired through the pressure transducer and strain gauges connected to a multichannel signal-conditioning amplifier (PCD-200A, Kyowa Dengyo Co., Tokyo, Japan) interfaced with a personal computer (PC-LM500J, NEC Inc., Tokyo, Japan), and recorded simultaneously with segmental displacement data.

\section{Experimental Protocol}


After testing the intact state, transection of the supraspinous and interspinous ligaments following a bilateral, partial facetectomy and partial diskectomy were performed at the L5-L6 level. For the partial facetectomy, the medial half of the facet joint and the ligamentum flavum were resected. The partial discectomy was defined as transection of the posterior longitudinal ligament and posterior part of the anulus fibrosus combined with removal of the nucleus pulposus. These destabilizations were chosen because discectomy have been demonstrated in the literature, and because sequential spinal instability can be created by these procedures. ${ }^{1}$ After the destabilization, spinal reconstructions were sequentially performed using pedicle screws with or without interbody fusion cages. The posterolateral fusion was simulated by pedicle screw fixations, and the PLIF by insertion of interbody cages: (1) L5-6 posterolateral fusion (one-segment posterolateral fusion) and (2) L5-6 posterolateral fusion with interbody fusion cages (one-segment PLIF/ posterolateral fusion). After testing one-segment fusion, destabilization was also performed at the L6-S1 level and three types of two-segment reconstruction were randomly assembled: (3) L5-6-S1 posterolateral fusion (two-segment posterolateral fusion), (4) L5-6-S1 posterolateral fusion with interbody fusion cages (two-segment PLIF/ posterolateral fusion), (5) L5-6-S1 posterolateral fusion in kyphosis (two-segment kyphotic posterolateral fusion). The order of this sequence was randomized among specimens. The Isola spinal system (screw, 6.25 x $45 \mathrm{~mm}$; rod, $6.35 \mathrm{~mm}$ in diameter; Depuy-AcroMed, Boston, MA) was used for pedicle screw fixation. For the interbody fusion, two Brantigan carbon cages $(10$ x 9 x $21 \mathrm{~mm}$; Depuy-AcroMed, Boston, MA) were used in each disc. For simulating the in situ posterolateral fusion, straight rods were used. For in situ PLIF/ posterolateral fusion, two cages were inserted into each disc and straight rods were used. For kyphotic posterolateral fusion, pre-bent rods were applied over the kyphosis (Figure 2). 
To standardize the compressive preload on the interbody cages, $125 \mathrm{~N}$ of compressive preload was applied to the specimen using an MTS Bionix 858 biaxial servohydrolic materials test machine (MTS Systems, Minneapolis, MN) while the rod-screw junctions were tightened. This preloading number was chosen after referring to a previous study involving intervertebral cages. ${ }^{16,18,21}$ The same biomechanical testing was repeated after each reconstruction.

\section{Statistical Analysis}

Statistical significance was determined using a repeated measurement analysis of variance (ANOVA) in addition to a post hoc multiple comparison using Fisher's Protected Least Significant Difference test (Fisher’s PLSD) at 95\% of confidence. 


\section{Results}

\section{Sagittal Alignment at the Reconstructed Segments}

The actual sagittal angle at the reconstructed segments in each specimen was measured on plain lateral radiographs by using the Cobb angle. The Mean angle ( \pm standard deviations) was $0.4 \pm 0.81^{\circ}$ lordosis in the intact group, $2.0 \pm 0.51^{\circ}$ lordosis in the one-segment posterolateral fusion group, $2.4 \pm 0.65^{\circ}$ lordosis in the one-segment PLIF/ posterolateral fusion group, $2.3 \pm 0.60^{\circ}$ lordosis in the two-segment posterolateral fusion group, $2.8 \pm 0.75^{\circ}$ lordosis in the two-segment PLIF/ posterolateral fusion group, and $29.0 \pm 0.75^{\circ}$ kyphosis in the two-segment kyphotic posterolateral fusion group. Excluding the kyphotic posterolateral fusion group, statistical differences were not detected between the other groups $(P>0.05)$.

\section{Range of motion of the Reconstructed Segments}

The ROM of the reconstructed segments demonstrated significant differences among the six groups under both flexion $(F=86.971, P<0.0001)$ and extension loading $(F=8.855, P<0.0001)$. A significant decrease was observed in the PLIF/ posterolateral fusion models compared with posterolateral fusion alone in both one and two segment fixation under flexion-extension loading. $(P<0.05)$. In the two-segment fusion models, both PLIF/ posterolateral fusion and posterolateral fusion procedures demonstrated a more restricted ROM than that of any of the one-segment fusion models, respectively (Figure 3).

\section{Intradiscal Pressure in the Adjacent Segment}

Statistical differences were detected between the six groups under flexion $(F=5.531, P=$ 0.0003) and extension ( $F=3.801, P=0.0051)$ loading. No statistical difference was observed 
between posterolateral fusion and PLIF/ posterolateral fusion procedures in one-segment fusion under the flexion-extension loading protocol $(P>0.05)$. Under flexion loading, two-segment PLIF/ posterolateral fusion model demonstrated the highest intradiscal pressure of all surgical treatment groups $(P<0.05)$. Under extension loading, both the two-segment PLIF/ posterolateral fusion and kyphotic posterolateral fusion groups demonstrated statistically higher intradiscal pressure than the one-segment fusion groups $(P<0.05)$. There were no significant differences between the two-segment posterolateral fusion and any of the one-segment models under flexion-extension loading $(P>0.05)$ (Figure 4).

\section{Lamina Strain in the Adjacent Segment}

Statistical differences were detected among the six reconstruction types in terms of flexion ( $F$ $=3.212, P=0.0131)$ and extension $(F=4.810, P=0.0010)$ strain in the adjacent segment. Similar to the intradiscal pressure results, statistical significance in the lamina strain was not found between the PLIF/ posterolateral fusion and posterolateral fusion procedures in the one-segment fusion model $\underline{(P>0.05)}$. Under flexion loading, the two-segment PLIF/ posterolateral fusion model revealed significantly higher lamina strain than the one-segment fusion models $(P<0.05)$. Under extension loading, a significant increase in the lamina strain was found in the two-segment PLIF/ posterolateral fusion and kyphotic posterolateral fusion groups compared to the one-segment fusion groups $(P<0.05)$. There were no significant differences between the two-segment posterolateral fusion and any of the one-segment models $\underline{(P>0.05)}$ (Figure 5). 


\section{Discussion}

\section{Background of the Study}

With the increase in the use of instrumentation for spinal fusion surgery, most surgeons have by now come to recognize that early breakdown of the adjacent segments can occur in some cases. Using a load controlled testing protocol, Oda et al ${ }^{18}$ created an in vitro spinal fusion model and demonstrated that additional interbody cages significantly increased construct stiffness and segmental motion at the adjacent segment compared with posterolateral fusion alone. Their study suggested that greater stiffness provided by instrumentation might accelerate degenerative changes within the adjacent motion segments.

Clinical studies have documented that lumbar fusion in a nonanatomic sagittal alignment could also cause degenerative changes at the adjacent segment. ${ }^{11,12}$ In an in vitro load controlled biomechanical study, Akamaru et al ${ }^{2}$ showed that flexion-extension motion at the segment above the fixation was increased when the fixed level was aligned in a hypolordotic fashion. These clinical and biomechanical researches emphasized the importance of sagittal realignment and maintenance of lordosis during fixation.

It has also been reported that reduction in the number of mobile segments increases the load in the adjacent unfused segments causing these early degenerative changes. ${ }^{8,17}$ Using a displacement controlled testing protocol, Nagata et $\mathrm{al}^{17}$ investigated the biomechanical effects of long thoraco-lumbar instrumentation on the remaining motion of adjacent segments They found that lumbosacral motion and facet loading were significantly increased after surgical immobilization and that the extent of the increase was dependent on the number of immobilized segments. In a retrospective clinical and radiographic study, Edwards et $\mathrm{al}^{8}$ examined patients with fused the 
thoracic spine to L5 and reported that subsequent advanced degeneration of the L5-S1 disc is common after such a long fusion, which is often requires further operations. Therefore, the surgeon is often posed with making such an important decision early in treatment to decide the most appropriate fusion level.

These aforementioned studies suggest that all of these parameters - construct stiffness, sagittal alignment, and the number of the fused segment - are important factors affecting the degenerative changes in the adjacent segments. There have been few reports, however, to investigate these interactions or synergistic effects of these factors. Particularly, no clinical or biomechanical studies have addressed these issues, in relation to the PLIF procedure. It remains undermined whether degenerative changes in the adjacent segment can be reduced by PLIF procedure, by restoring the sagittal alignment and achieving short segment fusion.

\section{Biomechanical Data and Clinical Relevance}

In this study, the ROM of the operative segments was significantly decreased in the PLIF/ posterolateral fusion models compared with posterolateral fusion alone, for both one and two segment fusion. For two-segment fusion procedures, both PLIF/ posterolateral fusion and posterolateral fusion demonstrated a reduced ROM than the one-segment fusion models. These results were consistent with the previous studies findings, which reported that intervertebral cages increased the construct stiffness ${ }^{3,18,21}$ and that immobilization of long segment instrumentation produced greater overall construct stiffness. ${ }^{17}$

There was no statistical difference in intradiscal pressure and lamina strain within the adjacent segment between one-segment fusion procedures. If the number of fused segments is increased, however, the pressure and strains were also increased more in the PLIF/ posterolateral fusion procedure than the posterolateral fusion procedure. These results suggest that there is no 
difference between any of the one-segment fusion procedures in terms of the biomechanical effects on the adjacent segment. However, if the fusion level is increased, the effects on the adjacent segment also become larger when using the PLIF/ posterolateral fusion procedure rather than the posterolateral fusion alone.

In the present study, one-segment PLIF/ posterolateral fusion model demonstrated a reduced intradiscal pressure and lamina strain compared to the kyphotic two-segment posterolateral fusion model in spite of higher levels of initial stiffness. These results suggest that if the number of fused levels can be reduced by correction of local kyphosis using the PLIF procedure, this surgical planning may be valuable for reducing degenerative changes within the adjacent segment. Furthermore, our findings suggest that the PLIF/ posterolateral fusion procedure with kyphotic deformity may have the worst biomechanical affects on the adjacent segment. As a general overview, it appears that the stiffer a segment is, the more one can expect to see increased intradiscal pressure and lamina strain in an adjacent segment. The one exception to this rule is if there is a kyphotic deformity. Hence, when the PLIF/ posterolateral fusion procedure is performed, the local kyphotic deformity must be corrected and fusion levels should be minimized.

\section{Study Limitations and Future Direction}

A calf spine model was used in this investigation. There are some differences in the anatomic characteristics between human and calf spines. The calf spine, however, was reported to exhibit similar mechanical responses to those of the human spine under a range of loading conditions. ${ }^{24,25}$ Furthermore, compared with human cadavers, small inter-specimen variability in size, bone mineral density, and age in the calf spine provide big advantages in consistency for the materials used in these biomechanical tests. ${ }^{3,18,21}$ On the other hand, the calf model does not represent the 
normal patient who would undergo such an operation in this study. The significant disc height (with a normal disc) at the fusion level is atypical as well. All the disc spaces were of normal height in the present model. In a human who undergoes surgery, the disc would not be at normal height nor would the adjacent levels. Many patients with loss of disc interspace height have a $\underline{\text { much more stable and stiff (in axial loading) ventral column. }}$

In the present study, pedicle screw fixation was used for the posterolateral fusion model. This procedure did not present precise posterolateral fusion because in posterolateral fusion, the fusion is across the transverse processes, not through the pedicle.

To evaluate the stability of the fused segment, we used a displacement gauge. The gauge could only detect linear displacement data and was not able to detect the location of the axis of rotation and real rotational motion, which might change under various spinal reconstructions. Therefore, only a rough estimate may be provided by comparing absolute displacement values for the same specimen.

In the current study, the sacrum was included for the fusion area in two segment fusion models. Using a human cadaveric spine, Untch et al ${ }^{23}$ showed that a statistically significant increase in L3-L4 motion occurred in flexion-extension loading in the L4-L5-S1 fusion model versus the L4-L5 model. The biomechanical difference at the cranial adjacent level might be easily detected in the present two segment fusion models than in typical two segment fusion models that did not include the sacrum.

The reconstruction models in this study addressed the immediate condition of the spinal construct after surgery. The effects of bony fusion on the adjacent segments, however, remain unclear. The present study could not detect the difference of biomechanical effects on the adjacent segment between one segment PLIF/ posterolateral fusion and posterolateral fusion groups, though PLIF/ posterolateral fusion model demonstrated statistically higher construct 
stiffness. However, the setting of the cages into the vertebrae over time may affect the stability of the motion segment. The results of a previous in vivo study demonstrated that solid osseous interbody fusion provided greater spinal construct stiffness. ${ }^{7}$ Additional clinical or animal studies are required to investigate the long-term effects of bony fusion on the adjacent segments. 


\section{References}

1) Abumi K, Panjabi MM, Kramer KM, Duranceau J, Oxland T, Crisco JJ: Biomechanical evaluation of lumbar spinal stability after graded facetectomies. Spine 15:1142-1147,1990

2) Akamaru T, Kawahara N, Tim Yoon S, Minamide A, Su Kim K, Tomita K, et al: Adjacent segment motion after a simulated lumbar fusion in different sagittal alignments. a biomechanical analysis. Spine 28:1560-1566,2003

3) Brodke DS, Dick JC, Kunz DN, McCabe R, Zdeblick TA: Posterior lumbar interbody fusion: A biomechanical comparison, including a new threaded cage. Spine 22:26-31,1997

4) Buttermann GR, Kahmann RD, Lewis JL, Bradford DS: An experimental method for measuring force on the spinal facet joint: Description and application of the method. J Biomech Eng 113:375-386,1991

5) Chow DH, Luk KD, Evans JH, Leong JC: Effect of Short Anterior Lumbar Interbody Fusion on Biomecanics of Neighboring Unfused Segments. Spine 5:549-555,1996

6) Cunningham BW, Kotani Y, McNulty PS, Cappuccino A, McAfee PC: The effect of spinal destabilization and instrumentation on lumbar intradiscal pressure. An in vitro biomechanical analysis. Spine 22:2655-2663,1997

7) Cunningham BW, Kanayama M, Parker LM, Weis JC, Sefter JC, Fedder IL, et al: Osteogenic protein versus autologous interbody arthrodesis in the sheep thoracic spine. Spine 24:509-518,1999

8) Edwards CC 2nd, Bridwell KH, Patel A, Rinella AS, Jung Kim Y, Berra AB, et al: Thoracolumbar deformity arthrodesis to L5 in adults: The fate of the L5-S1 disc. Spine 28:2122-2131,2003 
9) Heth JA, Hitchon PW, Goel VK, Rogge TN, Drake JS, Torner JC: A biomechanical comparison between anterior and transverse interbody fusion cages. Spine 26:e261-267,2003

10) Hitchon PW, Goel VK, Rogge TN, Torner JC, Dooris AP, Drake JS, et al: In vitro biomechanical analysis of three anterior thoracolumbar implants. J Neurosurg (Spine 2) 93: $252-258,2000$

11) Jackson RP, McManus AC: Radiographic analysis of sagittal plane alignment and balance in standing volunteers and patients with low back pain matched for age, sex, and size. Spine 19: $1611-1618,1994$

12) Kawakami M, Tamaki T, Ando M, Yamada H, Hashizume H, Yoshida M: Lumbar sagittal balance influences the clinical outcome after decompression and posterolateral spinal fusion for degenerative lumbar spondylolisthesis. Spine 27: 59-64,2002

13) Kumar MN, Baklanov A, Chopin D: Correlation between sagittal plane changes and adjacent segment degeneration following lumbar spine fusion. Eur Spine J 10:314-319,2001

14) Lee CK: Accelerated degeneration of the segment adjacent to a lumbar spine. Spine 13:375-377,1988

15) Lehmann TR, Spratt KF, Tozzi JE, Weinstein JN, Reinarz SJ, el-Khoury GY, et al: Long term follow-up of lower lumbar fusion patients. Spine 12:97-104,1987

16) Lund T, Oxland TR, Jost B, Cripton P, Grassmann S, Etter C, et al: Interbody cage stabilization in the lumbar spine: biomechanical evaluation of cage design, posterior instrumentation, and bone density. J Bone Joint Surg Br 80:351-359,1998

17) Nagata H, Schendel MJ, Transfeldt EE, Lewis JL: The effect of immobilization of long segments of the spine on the adjacent and distal facet force and lumbosacral motion. Spine 18:2471-2479,1993 
18) Oda I, Abumi K, Yu B-S, Sudo H, Minami A: Types of spinal instability that require interbody support in posterior lumbar reconstruction. Spine 28:1573-1580,2003

19) Oda I, Cunningham BW, Buckley RA, Goebel MJ, Haggerty CJ, Orbegoso CM, et al: Does Spinal Kyphotic Deformity Influence the Biomechanical Characteristics of the Adjacent Motion Segments? Spine 24:2139-2146,1999

20) Panjabi MM: Biomechanical evaluation of spinal fixation devices: I. A conceptual framework. Spine 13:1129-1133,1988

21) Sudo H, Oda I, Abumi K, Ito M, Kotani Y, Minami A: In vitro biomechanical effects of reconstruction on adjacent motion segment: comparison of aligned / kyphotic posterolateral fusion with aligned posterior lumbar interbody fusion/ posterolateral fusion. J Neurosurg (Spine

2) 99: $221-228,2003$

22) Suk SI, Lee CK, Kim WJ, Lee JH, Cho KJ, Kim HG: Adding Posterior Lumbar Interbody Fusion to Pedicle Screw Fixation and Posterolateral Fusion After Decompression in Spondylolytic Spondylolisthesis. Spine 22:210-219,1997

23) Untch C, Liu Q, Hart R: Segmental motion adjacent to an instrumented lumbar fusion. the effect of extension of fusion to the sacrum. Spine 29:2376-2381,2004

24) Wilke HJ, Krischak S, Claes L: Biomechanical comparison of calf and human spines. J Orthop Res 14:500-503,1996

25) Wilke HJ, Krischak ST, Wenger KH, Claes LE: Load-displacement properties of the thoracolumbar calf spine: experimental results and comparison to known human data. Eur Spine J 6:129-137,1997 


\section{Figure Legends}

Figure 1.

Experimental apparatus and measurement system. Pure moments were applied to the specimen with use of a system of weights and pulleys. Anterior segmental displacement was recorded using a displacement gauge. Intradiscal pressure and lamina strain within the superior adjacent segment were measured using a pressure needle transducer and uniaxial surface-strain gauges, respectively.

Figure 2.

A schematic diagram of five lumbar reconstruction models. Posterolateral fusion was simulated by the pedicle screw system and the PLIF by interbody fusion cages: a, L5-6 posterolateral fusion (one-segment PLF); b, L5-6 posterolateral fusion with interbody fusion cages (one-segment PLIF/PLF); c, L5-6-S1 posterolateral fusion (two-segment PLF); d, L5-6-S1 posterolateral fusion with interbody fusion cages (two-segment PLIF/PLF); e, L5-6-S1 posterolateral fusion of the kyphosis (two-segment kyphotic PLF).

\section{Figure 3.}

The mean range of motion (ROM) (and standard deviations) of the reconstructed segments from the intact spine and five reconstruction models under flexion- extension loading. All the data has been normalized for the initial, intact spine. 


\section{Figure 4.}

The mean intradiscal pressure (and standard deviations) within the superior adjacent segment of the intact spine and five reconstruction models under flexion-extension loading. All the data has been normalized for the initial, intact spine.

\section{Figure 5.}

The mean lamina strain (and standard deviations) within the superior adjacent segment for the intact spine and five reconstruction models under flexion-extension loading. All the data has been normalized for the initial, intact spine. 


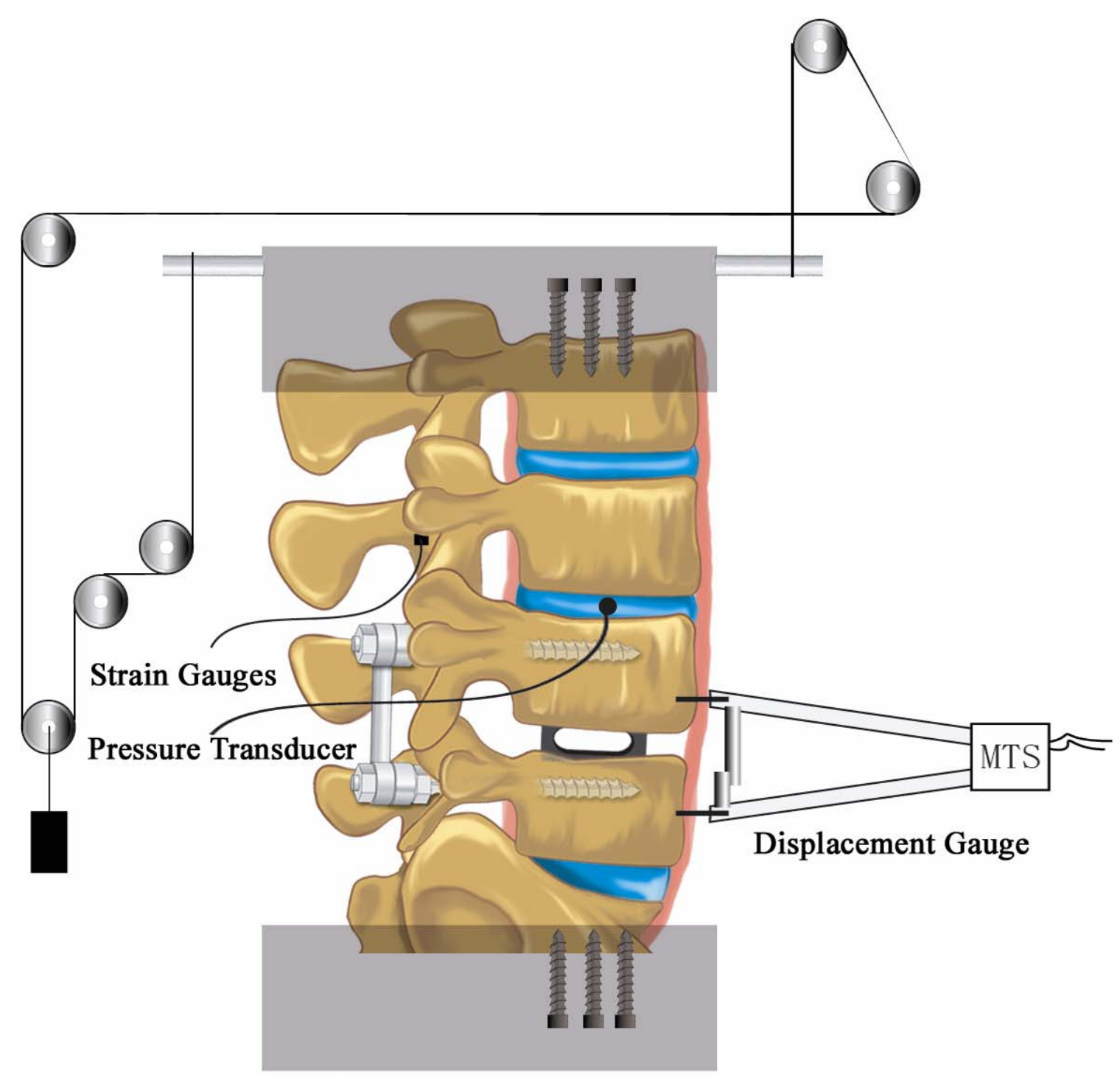




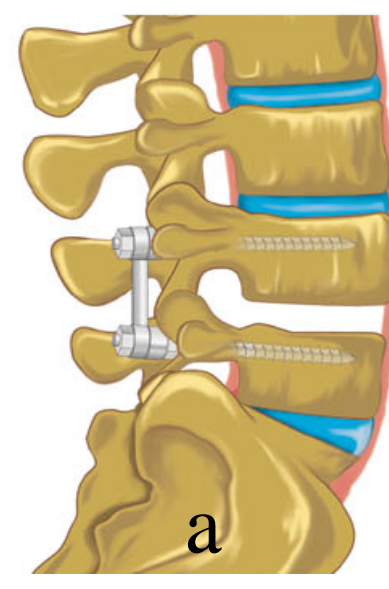

PLF

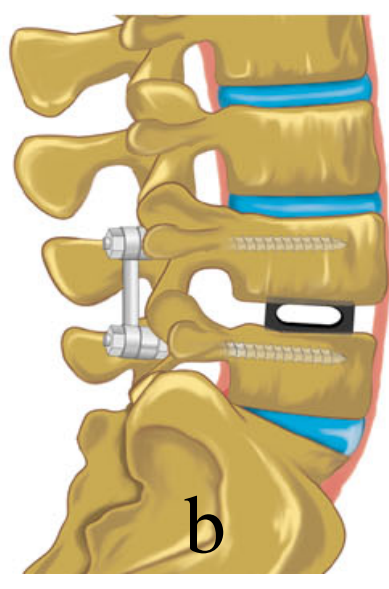

PLIF/PLF

One-segment fixation

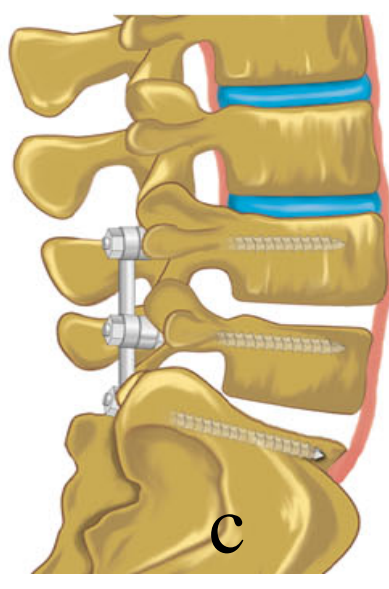

PLF

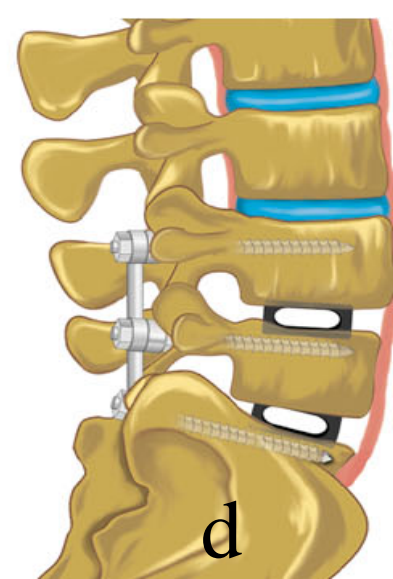

PLIF/PLF

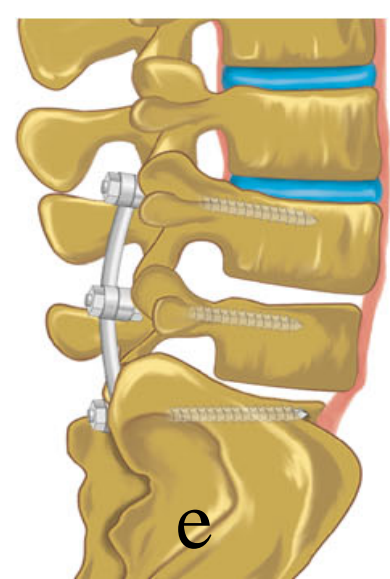

Kyphotic PLF

Two-segment fixation 


\section{Range of motion of Reconstructed Segments}

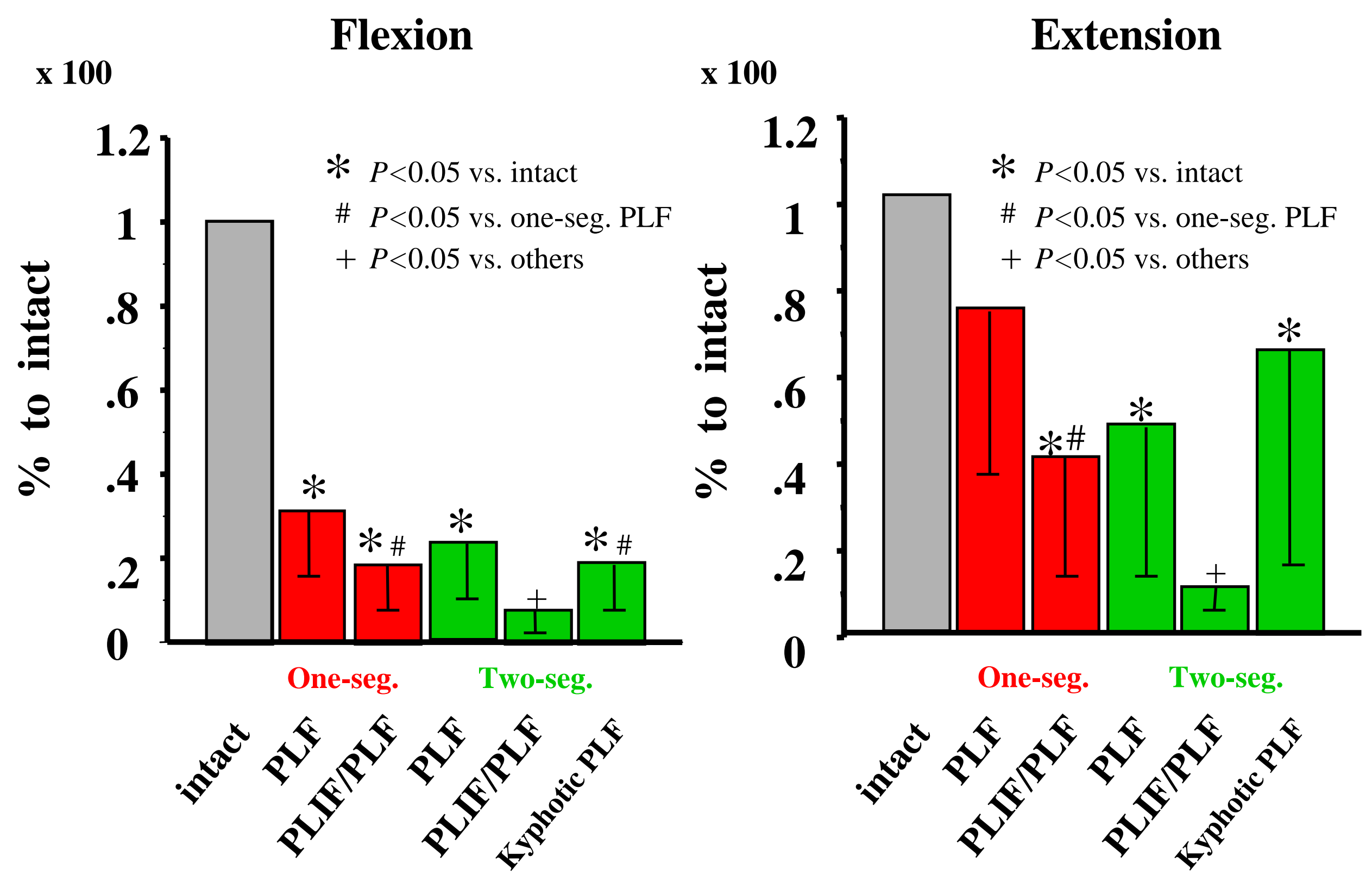




\section{Intradiscal Pressure in Adjacent Segment}

Flexion

* $P<0.05$ vs. intact
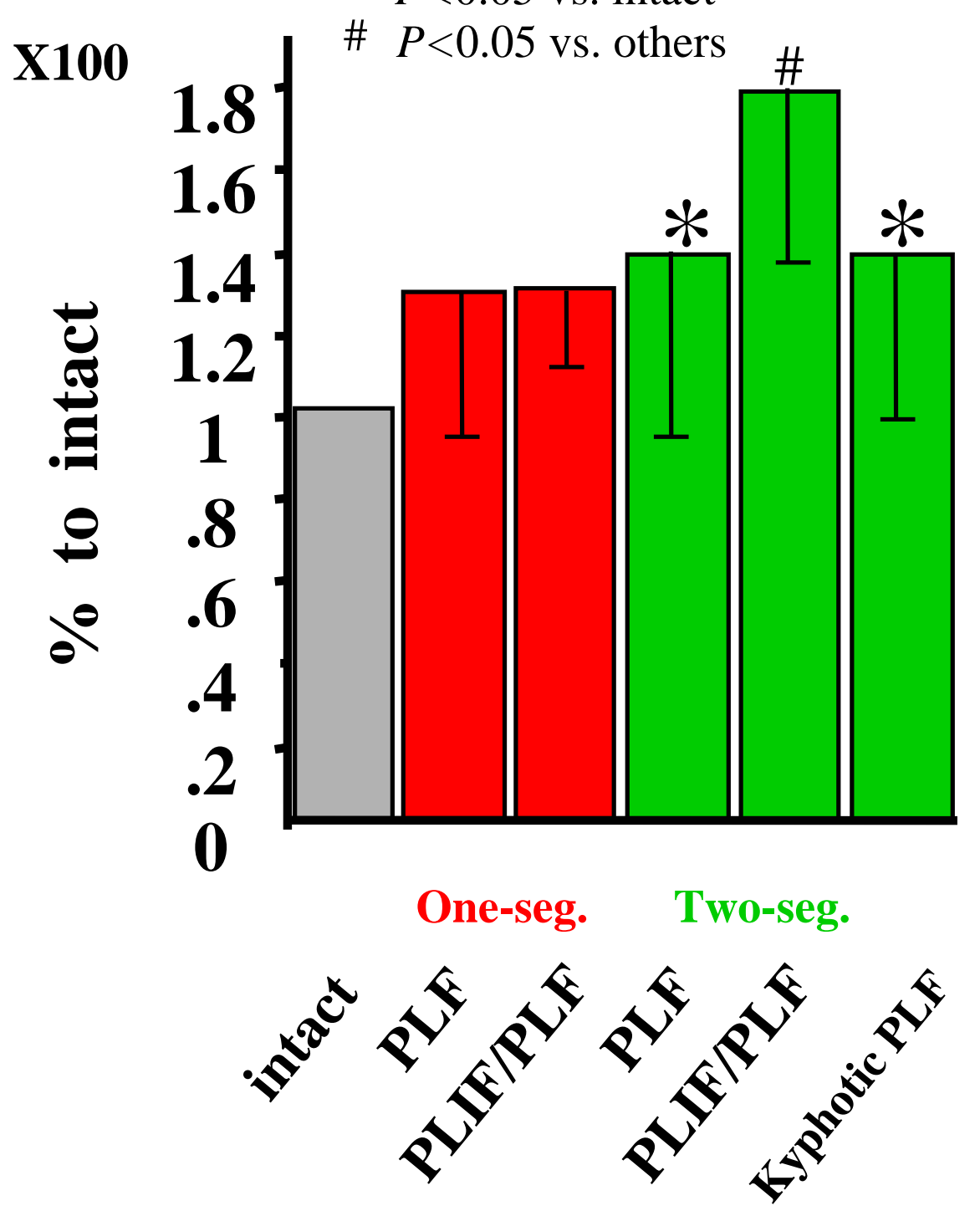

Extension
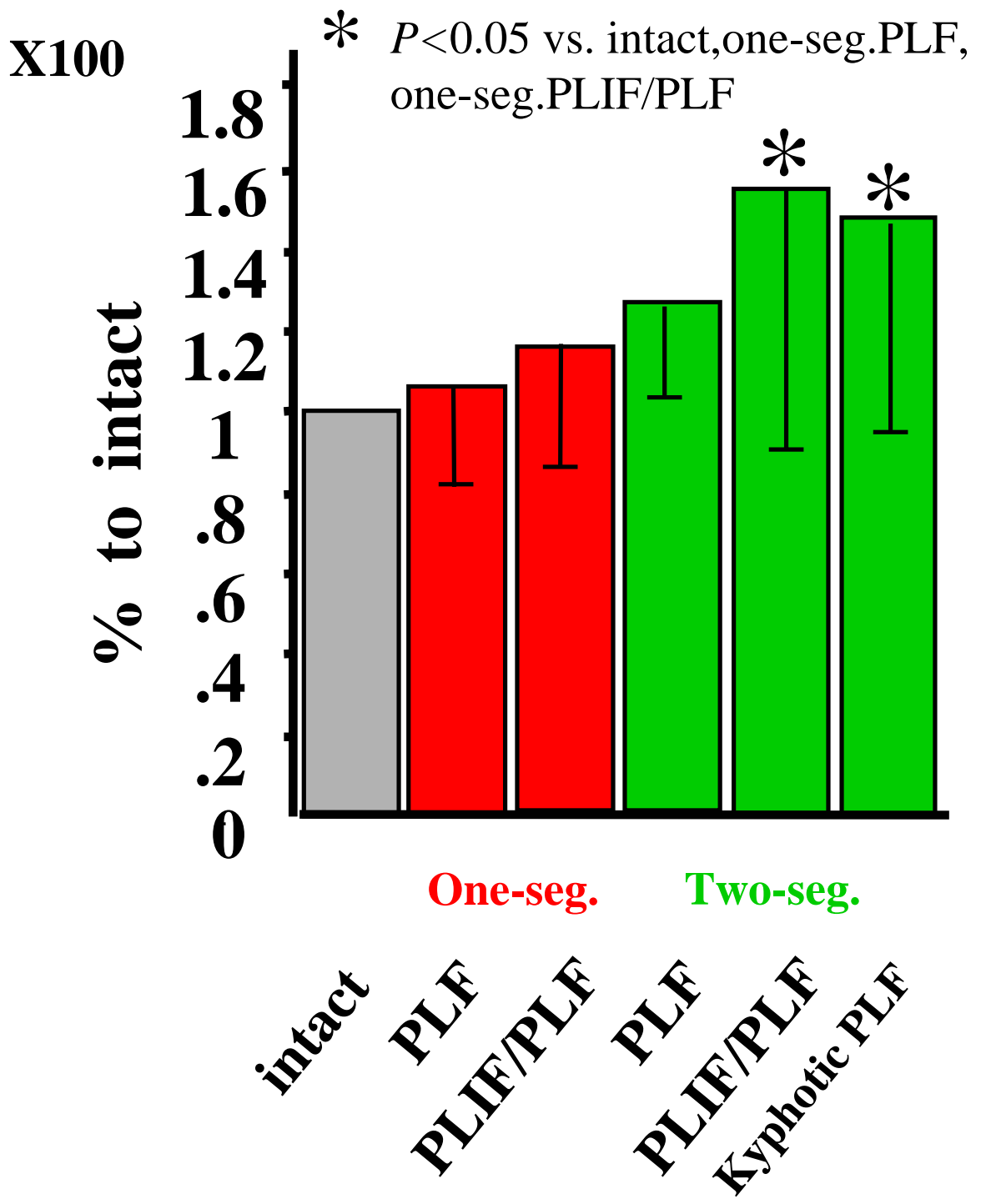


\section{Lamina Strain in Adjacent Segment}

\section{Flexion}
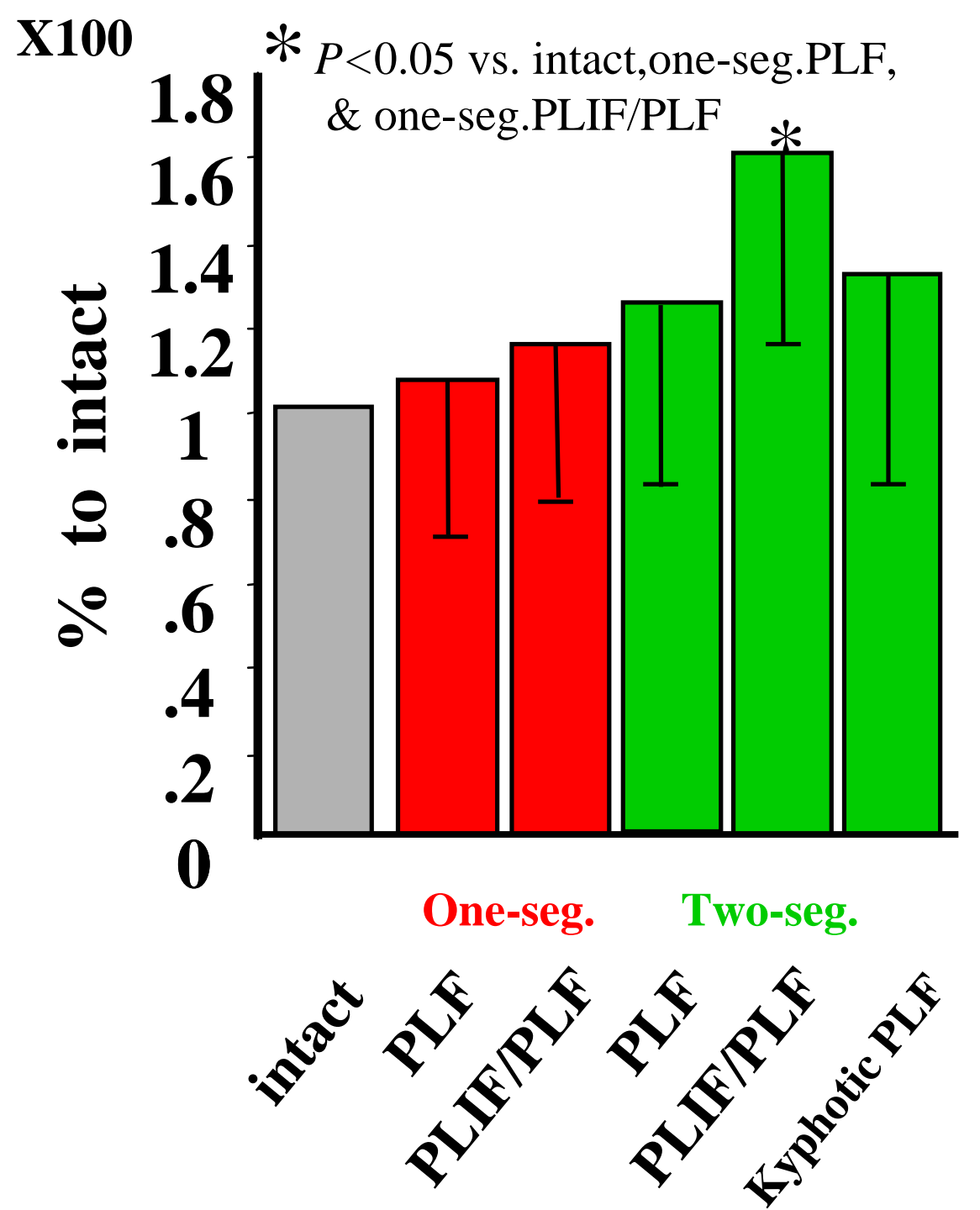

Extension

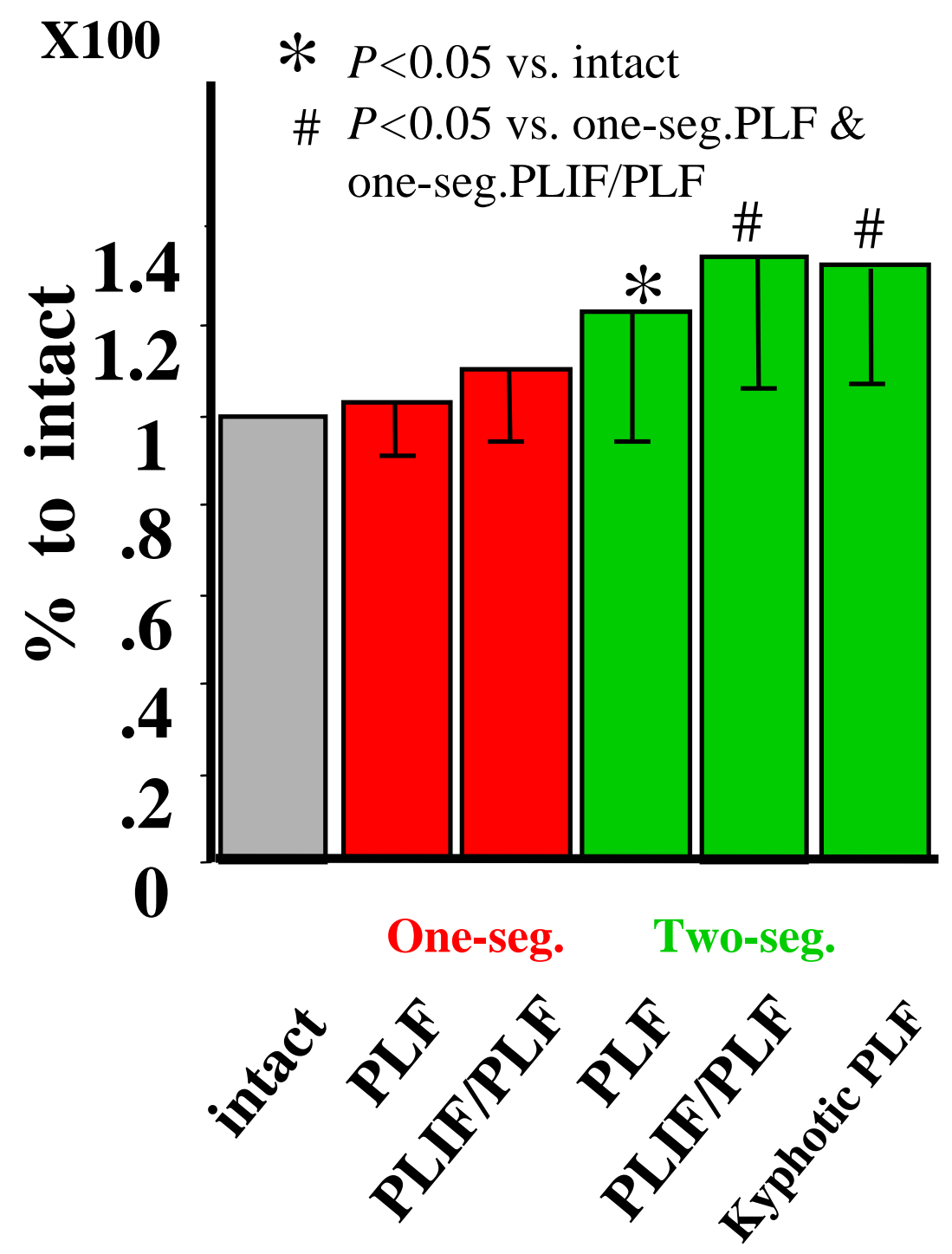

